\title{
CALIDAD DE VIDA LABORAL Y LA DISPOSICIÓN AL CAMBIO ORGANIZACIONAL EN FUNCIONARIOS DE EMPRESAS DE LA CIUDAD DE BOGOTÁ - COLOMBIA
}

\author{
Mónica García Rubiano*, Carlos Forero Aponte \\ Universidad Católica de Colombia
}

Recibido, junio 25/2014

Concepto evaluación, mayo 19/2015

Aceptado, octubre 19/2015

\begin{abstract}
Referencia: García Rubiano, M. \& Forero Aponte, C. (2016). Calidad de vida laboral y la disposición al cambio organizacional en funcionarios de empresas de la ciudad de Bogotá - Colombia. Acta Colombiana de Psicología, 19(1), 79-90. DOI: 10.14718/ ACP.2016.19.1.5
\end{abstract}

Resumen

El propósito de la investigación fue determinar la relación entre la calidad de vida laboral y la disposición al cambio organizacional en una muestra de 100 funcionarios de empresas de la ciudad de Bogotá. Se realizó bajo un diseño transversal correlacional, a través de la aplicación de dos instrumentos: el instrumento de medición de cambio organizacional (IMC) (García \& Forero, 2010) y el de calidad de vida percibida en organizaciones (Gómez - Rada, 2011). La participación de la población fue voluntaria. El análisis de los datos se realizó por medio del Rho de Spearman. Los resultados obtenidos muestran una correlación significativa al 0.001 con todas las variables de cambio organizacional y de calidad de vida, excepto las de resistencia al cambio. Estos hallazgos sugieren que algunos de los aspectos a resaltar son la importancia de la calidad de vida y el compromiso con las tareas a realizar, ya que estos definen las metas y objetivos planteados por la entidad y enmarcan el trabajo y la productividad de los empleados, al igual que su disposición al cambio.

Palabras Clave: cambio organizacional, calidad de vida laboral.

\section{QUALITY OF LIFE AT WORKAND WILLINGNESS TOWARD ORGANIZATIONAL CHANGE IN OFFICERS OF COMPANIES IN THE CITY OF BOGOTÁ - COLOMBIA}

\begin{abstract}
The purpose of the research was to determine the relationship between quality of work life and readiness to organizational change in a sample of 100 company workers in the city of Bogota. It was conducted following a correlational cross-sectional design through the application of two instruments: one that measures organizational change (BMI) (García \& Forero, 2010) and another one that assesses perceived quality of life in organizations (Gómez - Rada, 2011). The sample participation was voluntary and data were analyzed using Spearman's Rho. Results show a significant correlation at 0.001 in all the variables of organizational change and quality of life with the exception of resistance to change. This suggests that some of the aspects to be highlighted are the importance of quality of life and commitment to the tasks to be performed as these define the goals and objectives set by the entity and frame the work and productivity of employees as well as their readiness to change.

Key words: organizational change, quality of work life.
\end{abstract}

* Mónica García Rubiano y Carlos Forero Aponte, D.P: Av. Caracas No. 46 - 40. Tel. (57) 3277300 ext. 5050, mgarcia@ucatolica.edu. co,cforero@ucatolica.edu.co 


\title{
QUALIDADE DE VIDA PROFISSIONAL E A DISPOSIÇÃO À MUDANÇA ORGANIZACIONAL EM FUNCIONÁRIOS DE EMPRESAS DA CIDADE DE BOGOTÁ - COLÔMBIA
}

\author{
Resumo
}

\begin{abstract}
O propósito desta pesquisa foi determinar a relação entre a qualidade de vida profissional e a disposição à mudança organizacional numa amostra de 100 funcionários de empresas da cidade de Bogotá. Realizou-se sob um desenho transversal correlacional por meio da aplicação de dois instrumentos: o instrumento de medição de mudança organizacional (García e Forero, 2010) e o de qualidade de vida percebida em organizações (Gómez-Rada, 2011). A participação da população foi voluntária. A análise dos dados foi realizada por meio do rho de Spearman. Os resultados obtidos mostram uma correlação significativa a 0.001 com todas as variáveis de mudança organizacional e de qualidade de vida, exceto as de resistência à mudança. Esses achados sugerem que alguns dos aspectos a ressaltar são a importância da qualidade de vida e do compromisso com as tarefas a realizar visto que estes definem as metas e os objetivos propostos pela entidade e determinam o trabalho e a produtividade dos empregados, assim como sua disposição à mudança.

Palavras-chave: mudança organizacional, qualidade de vida profissional.
\end{abstract}

\section{INTRODUCCIÓN}

Brindar un ambiente laboral propicio es un tema de suma importancia para cualquier organización. El talento humano es la fuerza que mueve cualquier proceso, facilita el desarrollo del mismo y, sin duda alguna, fortalece el progreso continuo tanto de los empleados como de la organización. Es relevante no solo suplir las necesidades básicas del empleado; de por medio aparece una gran variedad de necesidades personales que influyen directamente en los comportamientos y actitudes que el trabajador tiene dentro de la organización; por esto dichas necesidades deben ser ajustadas dentro de la empresa para que la calidad de vida laboral del empleado sea óptima (Nair, 2013).

Elizur y Shye (1990) señalan que la calidad de vida laboral (CVL) se ha estudiado desde dos perspectivas teórico - metodológicas: a) la calidad de vida en el entorno del trabajo, cuya meta es conseguir mejorar la calidad de vida mediante el logro de los intereses organizacionales. Su interés es analizar la organización como un sistema, teniendo en cuenta los diferentes subsistemas que la componen; de esta forma pretende alcanzar una mayor productividad y eficacia organizacionales como paso previo sin el cual no sería posible satisfacer las demandas de cada trabajador y, b) la calidad de vida psicológica, orientada a mostrar un mayor interés por el trabajador, desarrollando análisis que permitan establecer los elementos puntuales que afectan el bienestar del mismo en su vida cotidiana. Aunque ambos enfoques tienen como meta común mejorar la vida laboral, discrepan en los objetivos que persiguen.

Desde estas dos perspectivas, González, Peiró y Bravo (1996) exponen que la CVL da cuenta de la forma en que se produce la experiencia laboral, tanto en sus condiciones: a) objetivas, en donde se evalúa de manera global toda la organización como un sistema en todos los niveles, teniendo en cuenta características físicas y ambientales del puesto de trabajo que pueden dar lugar a una mayor o menor calidad de vida laboral, como b) subjetivas, desde el punto de vista del trabajador, considerando los elementos que influyen directamente en la forma como percibe su trabajo, entendiendo las características individuales que hacen que se creen perspectivas alrededor del trabajo, las cuales incluyen todas aquellas características emocionales y actitudinales que puedan influir .

La calidad de vida laboral es entendida por Pérez, Campos, Negro y Caballero (2011) como la percepción entre las exigencias del trabajo y los medios disponibles para lograr dichas demandas, las cuales pueden provenir de manera directa del trabajador o de la organización. Este concepto contiene elementos subjetivos y objetivos: los subjetivos hacen referencia a las creencias que desarrolla el trabajador y que se originan a partir de las realidades laborales; en este sentido, se afirma que las experiencias subjetivas no son una dimensión de la vida sino la vida misma. Por otra parte, los elementos objetivos son aquellos que constituyen el medio ambiente laboral, como las condiciones de trabajo, el salario, la salud, etc. (Segurado \& Argulló, 2000).

La percepción que tienen los empleados de los elementos subjetivos y objetivos puede llevarlos a asumir diversas posiciones frente a procesos de cambio que se estén gestando en las organizaciones. Según García, Camargo, Cervera, Ramírez y Romero (2010), al ser las organizaciones sistemas abiertos y dinámicos, estas cambian de manera constante; por lo tanto, es necesario entender en qué consistente el cambio, cuáles son los factores que influyen en este y la manera más apropiada de guiarlo en la organización. 
El cambio organizacional no se debe entender como un elemento aislado al interior de las organizaciones; por el contrario, debe ser visto como un elemento dinamizador que permite ser abordado desde diferentes aspectos del comportamiento organizacional en sus tres niveles (individuo, grupo y organización). Al respecto, esta investigación corresponde a una línea de trabajo en donde se han desarrollado estudios, tanto a nivel individual como grupal. A nivel del individuo, con variables como actitud (García, Rojas \& Díaz, 2011), motivación (García, Gómez \& Londoño, 2009), motivación y satisfacción (García \& Forero, 2014) y justicia organizacional (García \& Forero, 2014); a nivel grupal se han estudiado variables como liderazgo (García, 2011), comunicación (García, Arias \& Gómez, 2013) y trabajo en equipo (García, Camargo, Cervera, Ramírez \& Romero, 2010). En términos generales, los estudios mencionados muestran correlaciones con las diferentes variables mencionadas, lo que permite suponer aspectos interrelacionales en las organizaciones y, por tanto, su influencia en los procesos de cambio.

El cambio organizacional es entendido por Acosta (2004) como un conjunto de transformaciones que se producen por fuerzas internas o externas a las organizaciones, y que generalmente buscan incrementar la efectividad de las mismas (Freire \& Gutiérrez-Rubí, 2010).

En lo que respecta a las fuerzas que generan un cambio, estas pueden ser: a) estructurales, o referentes a fusiones y adquisiciones; b) reducción de costes, lo cual hace alusión a la eliminación de actividades no esenciales o a la supresión de otros costos en las operaciones; c) cambio de proceso, cuyo énfasis está en hacer que este sea más eficaz y menos costoso; y d) cambio cultural, el cual se centra en el aspecto humano, como lo es el cambio de una dirección de control a una dirección participativa (Luecke, 2003).

Por su parte, Palací, (2005), diferencia entre cambio planeado y cambio no planeado. El primero apunta a la consecución de metas, es más organizado y tiene más control y relación con temas financieros como los costos que se generan. El segundo hace referencia a las acciones que se generan como respuesta a la exigencia de variables externas tales como nuevos direccionamientos o exigencias del mercado. El cambio involucra innovaciones en los procesos, servicios, productos, en la estructura y la dirección de la empresa.

El modelo de cambio planeado propuesto por Lippitt, Watson y Westley (1958) es uno de los más representativos del cambio, el cual señala que toda la información debe ser compartida en toda la organización y que esta solo será funcional si se traduce en planes de acción para mejoramiento en la empresa. Las fases del modelo son la de explorar la necesidad, definir la entrada de un agente de cambio preferiblemente externo con el fin de realizar un diagnóstico y planear metas y soluciones para generar acciones reales y posteriormente lograr estabilizar y evaluar la organización (Citados por Hernández, Gallarzo y Espinosa, 2011).

Según Ruiz, Ruiz, Martínez \& Peláez (1999) hay que tener en cuenta la diferenciación entre los factores del cambio organizacional y la gestión del cambio organizacional. Los factores del cambio organizacional son aquellos que, por diversas razones, una organización puede detectar la necesidad de cambiar, y comprenden aspectos de orden tecnológico, estructural, de personal y cultural. En el mundo actual de las organizaciones, caracterizado por la globalización, el poder de los clientes y la avalancha de la información, estas deben estar en un proceso permanente de cambio para lograr un mejoramiento continuo. Son los factores tecnológicos, estructurales, culturales y del personal, de los que las organizaciones disponen para poder actuar sobre ellos y, de esta forma, conseguir la adaptación y/o proactividad que necesitan. Para gestionar el cambio en estos factores se han de tener muy claro dos aspectos: las fases del cambio y los elementos para la gestión del cambio. Se considera que el cambio organizacional se puede estructurar en cinco fases: detectar la necesidad de cambio, realizar el diagnóstico de la situación actual, llevar a cabo la planificación de acciones, instaurar el cambio y llevar a cabo el control y evaluación de los cambios a medida que estos se van produciendo.

Es importante resaltar que la forma como se maneje el cambio va a generar mayor o menor resistencia. Esta es define como en un desacuerdo organizacional con respecto al cambio, ya que muchos individuos lo consideran desagradable o no lo entienden, bien sea porque no ha sido explicado de una manera clara o porque se genera incertidumbre frente al proceso (Vicenzi, Melo \& Fonseca, 2011).

Al respecto, la literatura tiene una serie de debates sobre la forma en que se debe llevar a cabo el cambio organizacional, pero siempre coinciden en la idea de que se debe instaurar enfocado en que los miembros de la organización reconozcan la necesidad de dicho proceso. De esta forma, la capacidad de cambio implica un enfoque sistémico para el desarrollo de la organización, aprovechando así la capacidad natural de las personas, sus percepciones respecto a la equidad en relación al trato y al estatus en el proceso, y los equipos de trabajo que deseen cambiar y apoyar el cambio, lo cual es un aspecto fundamental (Kerber \& Buono, 2010).

En un estudio realizado por Ernst, Lewis y Hammer (2010) se hace una revisión de las perspectivas de los empleadores en la vida laboral y sus iniciativas en relación 
con posibles fenómenos de cambio organizacional frente a dos retos: el primero hace referencia a la organización estructural (trabajo flexible, políticas de recursos humanos), y el segundo se refiere a factores culturales (supervisores de apoyo, clima). Los autores señalan que aunque la vida laboral y las iniciativas responden al propósito de satisfacer las necesidades de adaptación de la organización a los relaciones cambiantes entre el trabajo y la vida familiar y personal de los individuos, estos aspectos son marginados, en lugar de involucrarse en los sistemas de la organización; por lo tanto, la visión debe cambiar e integrarse para mejorar la eficacia de la organización.

Una investigación realizada por Gómez (2010) se propuso como objetivo conocer la apreciación que tenían los empleados temporales del Valle de Aburrá frente a ciertos factores del cambio organizacional y la influencia de este en su calidad de vida. El autor expone una estrategia para el mejoramiento de la calidad de vida en general, la cual se estructura en seis pasos: preparación, planificación, divulgación, despliegue, instauración y mejoramiento constante de la calidad y el cambio organizacional que este conlleve. La investigación concluyó que la calidad de vida en el trabajo requiere cambios: en la forma de ver y de hacer las labores, en la manera de llevarlas a cabo; en la manera de conducir la organización; en las variantes de participación de las personas, y en el contexto de las responsabilidades, entre otros. Esto no significa que sea necesario cambiarlo todo, sino que el progreso en materia de calidad demanda adecuaciones en diferentes aspectos de la administración que constituyen un obstáculo para su logro. Esto conlleva la necesidad de promover acciones que impulsen el desarrollo local sostenible, entendido como una responsabilidad compartida por todos y cada uno de los agentes sociales, económicos y medioambientales, los cuales, de una forma $\mathrm{u}$ otra, tienen algo que proponer y recomendar para mejorar continuamente la calidad de vida de los trabajadores.

De esta forma, la calidad de vida en el trabajo se convierte en un factor transversal para la toma de decisiones frente a los cambios que se deben realizar dentro de la organización; estos cambios deben fortalecer las dimensiones de los trabajadores en pro del desarrollo, la productividad y la eficacia de la organización.

En general, la influencia de la calidad de vida laboral y el cambio organizacional no han sido muy estudiados. Se prevé algún tipo de relación en cuanto a que la calidad de vida laboral constituye una percepción subjetiva del cambio organizacional, que involucra la forma como las personas asumen el proceso de cambio al interior de las organizaciones. Por ello, este estudio solo pretende explorar la posible relación entre las variables objeto de estudio y brindar referentes empíricos para futuras investigaciones.

De esta manera, teniendo en cuenta lo anterior, se genera la siguiente pregunta de investigación: ¿Cuál es la relación entre la calidad de vida laboral y la disposición al cambio organizacional en los funcionarios de empresas de la ciudad de Bogotá? Como objetivo general se plantea: Identificar la relación entre calidad de vida laboral y la disposición al cambio organizacional en funcionarios de empresas de la ciudad de Bogotá. Y como objetivos específicos: a) identificar la disposición al cambio organizacional en funcionarios de empresas de la ciudad de Bogotá y, b) identificar las condiciones de calidad de vida laboral en los funcionarios de empresas de la ciudad de Bogotá. De acuerdo con lo anterior, se planteó la siguiente hipótesis de investigación: Se presenta una relación significativa entre calidad de vida y cambio organizacional

\section{MÉTODO}

\section{Tipo de estudio}

Dado que las variables a investigar ya estaban presentes en los individuos y las organizaciones, y que no era factible realizar manipulación de las mismas, se optó por escoger un enfoque no experimental, con un estudio transversal de carácter correlacional, en donde se buscó establecer la relación entre las variables de cambio organizacional y calidad de vida, pero no se intentó determinar la causalidad entre las mismas (Hernández, Fernández \& Baptista, 2010). Con esta decisión metodológica se buscó establecer la relación entre las variables a partir de la información obtenida mediante la implementación de pruebas estandarizadas. Se justifica el desarrollo de este tipo de investigación dado que las variables cambio y calidad de vida ya estaban presentes en el contexto y no se buscaba realizar ningún tipo de manipulación respecto a las mismas, pero sí determinar las posibles relaciones que se presentan para contribuir con la comprensión del cambio organizacional.

\section{Participantes}

La investigación realizada fue correlacional, dado que se presentan variables de corte cuantitativo que están presentes en los individuos, previa la ejecución de la misma y no requiere de la realización de muestreos probabilísticos. Se evaluó la normalidad de las distribuciones para determinar el uso específico del coeficiente de correlación que en este caso correspondió al Rho de Spearman. El muestreo fue no probabilístico autoselectivo (Coolican, 2005); se escogieron 101 funcionarios de empresas en proceso de cambio de estructura, funciones y/o administración de la ciudad de Bogotá. Las condiciones para la selección de 
los participantes incluyeron que debían ser personas con tiempo de vinculación igual o superior a tres meses en la organización y aceptación voluntaria para participar en la investigación. Socio demográficamente la muestra estuvo compuesta fundamentalmente por mujeres $(52,5$ $\%$ ); el 74,3\% del total eran profesionales, y el 18,8\% eran técnicos; $55,4 \%$ eran soltero(a)s y $33,7 \%$ casado(a)s; y en cuanto al tipo de contrato, el $88,1 \%$ tenían contrato de prestación de servicios. El tiempo medio de vinculación era de 12,5 años y de antigüedad en el cargo de 8,3 años; tanto el tiempo de vinculación como el tiempo en el cargo presentan un sesgo positivo, lo que indica la tendencia a valores inferiores al promedio (asimetrías de 4,3 y 2,5 respectivamente).

Procedimiento

Para el desarrollo de esta investigación se tuvieron en cuenta las siguientes fases: a) envío de propuestas a diversas instituciones para realizar la aplicación; b) después de la aceptación por parte de las entidades, se establecieron los aspectos logísticos para realizar la aplicación de los instrumentos, iniciando por la conformación de los grupos poblacionales. Se dieron las instrucciones de confidencialidad y se realizó la aplicación colectiva de los instrumentos. La participación de los sujetos fue voluntaria, anónima y no se proporcionaron incentivos para responder los cuestionarios. El tiempo aproximado para diligenciar los instrumentos fue de hora y media.

Instrumentos

Para el desarrollo de esta investigación se empleó el Instrumento de Medición del Cambio - IMC- (García \& Forero, 2010). El instrumento está constituido por nueve variables principales: a) adaptabilidad, b) liderazgo, c) participación/integración, d) comunicación, e) trabajo en equipo, e) aceptación , f) resistencia al cambio, g) conocimiento de la organización, y h) cultura de cambio; por tres factores de primer orden: a) factor individual, b) factor grupal, y c) factor organizacional; y por dos factores de segundo orden a) factor I: gestión del cambio, y b) factor II: proyección y desarrollo del cambio.

La confiabilidad total de la prueba con base en el Alfa de Cronbach fue de 0,86. En la tabla 1 se presentan los niveles de confiabilidad para cada componente de la prueba IMC y las puntuaciones máximas; la puntuación mínima teórica para cada uno de los componentes es 1 .

Tabla 1.

Confiabilidad por Alfa de Cronbach y puntuaciones máximas para los distintos aspectos evaluados por IMC

\begin{tabular}{|c|c|c|c|c|}
\hline & Variable & $\begin{array}{l}\text { Cantidad de } \\
\text { Ítems }\end{array}$ & Alfa De Cronbach & $\begin{array}{l}\text { Puntuación } \\
\text { Máxima }\end{array}$ \\
\hline \multirow{9}{*}{ Variables generales } & Adaptabilidad & 5 & 0,512 & 20 \\
\hline & Liderazgo & 6 & 0,776 & 24 \\
\hline & Participación-Integración & 5 & 0,777 & 20 \\
\hline & Comunicación & 5 & 0,709 & 20 \\
\hline & Trabajo en equipo & 4 & 0,743 & 16 \\
\hline & Aceptación & 8 & 0,542 & 32 \\
\hline & Resistencia al cambio & 11 & 0,879 & 44 \\
\hline & Conocimiento de la organización & 4 & 0,604 & 16 \\
\hline & Cultura de cambio & 3 & 0,77 & 12 \\
\hline \multirow{3}{*}{$\begin{array}{l}\text { Factores de primer } \\
\text { orden }\end{array}$} & Individual & 11 & 0,745 & 44 \\
\hline & Grupal & 14 & 0,845 & 56 \\
\hline & Organizacional & 26 & 0,756 & 104 \\
\hline \multirow{2}{*}{$\begin{array}{l}\text { Factores de segun- } \\
\text { do orden }\end{array}$} & Gestión de cambio & 16 & 0,89 & 64 \\
\hline & Proyección y desarrollo del cambio & 6 & 0,736 & 24 \\
\hline Total & IMC & 51 & 0,86 & 204 \\
\hline
\end{tabular}


Para evaluar la calidad de vida laboral, se empleó la prueba que lleva el mismo nombre y fue diseñada por Gómez-Rada (2011), la cual consta de ocho dimensiones: Compensación y beneficios (5 ítems), Condiciones del ambiente y del trabajo (6 ítems), Naturaleza de la tarea (6 ítems), Desarrollo y seguridad laboral (3 ítems), Democracia organizacional (5 ítems), Derechos fundamentales (4 ítems), Equilibrio laboral (4 ítems), e Impacto social (6 ítems). La prueba empleada tiene un total de 39 ítems y consiste en una escala tipo Likert con valor mínimo de 1 y máximo de 4 por ítem; tiene una confiabilidad de 0.97 obtenida con Alfa de Cronbach. Se utilizó esta prueba dada la consistencia de la misma con la postura de Walton (1975, citado por Toro, 1991).

\section{RESULTADOS}

Para el análisis de resultados se obtuvieron los estadísticos descriptivos (medidas de tendencia central, dispersión, asimetría y curtosis), se determinó el comportamiento de las distribuciones mediante Kolmogorov-Smirnov y, finalmente se realizaron las correlaciones correspondientes mediante el coeficiente Rho de Spearman.

En la primera parte de los resultados, se presentan los análisis descriptivos de las variables disposición al cambio organizacional y calidad de vida laboral; y en la segunda, las correlaciones obtenidas con el coeficiente de correlación de Spearman. Este método se utilizó dado que al realizar análisis de normalidad de Kolmogorov-Smirnov se encontró que los datos no estaban normalmente distribuidos (Pérez, 2001).

Tabla 2.

Estadísticos descriptivos de las variables y factores del IMC

\begin{tabular}{|c|c|c|c|c|c|c|c|}
\hline & \multirow[t]{2}{*}{ Variables/Factores } & \multicolumn{6}{|c|}{ Estadísticos descriptivos } \\
\hline \multirow{11}{*}{ Variables generales } & & Media & Mediana & Moda & $\begin{array}{l}\text { Desv. } \\
\text { típ. }\end{array}$ & Asimetría & $\begin{array}{l}\text { Curto- } \\
\text { sis }\end{array}$ \\
\hline & Adaptabilidad & 18,54 & 20,00 & 20,00 & 2,41 & $-1,75$ & 2,34 \\
\hline & Aceptación & 26,20 & 27,00 & 29,00 & 3,06 & $-1,37$ & 1,87 \\
\hline & Comunicación & 17,31 & 18,00 & 20,00 & 3,14 & $-1,32$ & 1,67 \\
\hline & $\begin{array}{c}\text { Conocimiento de la orga- } \\
\text { nización }\end{array}$ & 13,81 & 15,00 & 16,00 & 2,66 & $-1,37$ & 1,84 \\
\hline & Cultura de cambio & 10,42 & 12,00 & 12,00 & 2,16 & $-1,55$ & 2,33 \\
\hline & Liderazgo & 22,06 & 24,00 & 24,00 & 2,93 & $-1,98$ & 4,50 \\
\hline & Participación Integración & 18,04 & 19,00 & 20,00 & 2,56 & $-1,33$ & 1,38 \\
\hline & Resistencia al cambio & 30,25 & 31,00 & 27,00 & 5,85 & $-0,36$ & 0,33 \\
\hline & Trabajo en equipo & 13,91 & 15,00 & 16,00 & 2,55 & $-1,43$ & 2,04 \\
\hline & Total & 170,53 & 176,00 & 178,00 & 17,55 & $-1,76$ & 2,92 \\
\hline \multirow{3}{*}{$\begin{array}{l}\text { Factores de primer } \\
\text { orden }\end{array}$} & Nivel Grupal & 49,26 & 52,00 & 56,00 & 7,28 & $-1,58$ & 2,97 \\
\hline & Nivel Individual & 40,60 & 44,00 & 44,00 & 5,00 & $-1,95$ & 4,19 \\
\hline & Nivel Organizacional & 80,67 & 83,00 & $83,00 \mathrm{a}$ & 8,15 & $-1,11$ & 0,85 \\
\hline \multirow{2}{*}{$\begin{array}{l}\text { Factores de segun- } \\
\text { do orden }\end{array}$} & $\begin{array}{l}\text { Gestión del cambio (Factor } \\
\text { de segundo Orden) }\end{array}$ & 58,21 & 61,00 & 64,00 & 6,84 & $-2,08$ & 5,46 \\
\hline & $\begin{array}{l}\text { Proyección y desarrollo del } \\
\text { cambio (Segundo Orden) }\end{array}$ & 21,01 & 23,00 & 24,00 & 3,60 & $-1,47$ & 1,68 \\
\hline
\end{tabular}


Respecto al IMC, en la tabla 2 se puede observar que, en genera, los promedios de las variables tienden a los valores más altos de la escala, llegando a encontrarse medianas en el máximo tope de la distribución (Adaptabilidad, Cultura de Cambio y Liderazgo). Los sesgos de las distribuciones pueden apreciarse en los valores de asimetría, destacándose que son negativas, lo que en conjunto con los demás valores de la distribución, evidencia la tendencia a ubicarse en las puntuaciones más altas. La única variable que presentó comportamiento normal fue resistencia al cambio. Por su parte las curtosis en general presentan valores positivos y, en conjunto con las desviaciones presentadas, evidencian la tendencia a formar grupos homogéneos. Estos aspectos pueden deberse a elementos de deseabilidad en los respondientes o a dificultades en la discriminación por parte del instrumento.

Tabla 3.

Estadísticos descriptivos de las dimensiones del instrumento Calidad de Vida Laboral

\begin{tabular}{ccccccc}
\hline \multicolumn{1}{c}{ Dimensiones } & \multicolumn{7}{c}{ Estadísticos descriptivos } \\
\hline & Media & $\begin{array}{l}\text { Medi- } \\
\text { ana }\end{array}$ & Moda & $\begin{array}{l}\text { Desv. } \\
\text { típ. }\end{array}$ & Asimetría & $\begin{array}{l}\text { Curto- } \\
\text { sis }\end{array}$ \\
Compensación y beneficios & 16,61 & 18,00 & 20,00 & 4,10 & $-1,32$ & 0,65 \\
Condiciones del ambiente y del trabajo & 21,16 & 22,00 & 24,00 & 3,39 & $-1,72$ & 2,87 \\
Naturaleza de la Tarea & 21,33 & 22,00 & 24,00 & 3,28 & $-1,96$ & 4,25 \\
Desarrollo y Seguridad Laboral & 10,17 & 11,00 & 12,00 & 2,25 & $-1,37$ & 1,18 \\
Democracia Organizacional & 17,35 & 19,00 & 20,00 & 3,49 & $-1,53$ & 1,51 \\
Derechos Fundamentales & 14,14 & 15,00 & 16,00 & 2,43 & $-1,66$ & 2,23 \\
Equilibrio Laboral & 13,67 & 15,00 & 16,00 & 2,78 & $-1,43$ & 1,13 \\
$\quad$ Impacto social & 21,77 & 23,00 & 24,00 & 3,11 & $-2,10$ & 4,55 \\
\hline
\end{tabular}

Al igual que el IMC, el instrumento de calidad de Vida Laboral también mostró que las dimensiones evaluadas no se comportaban normalmente. Al comparar los promedios de las dimensiones con las puntuaciones máximas, se encuentra que tienden a estas $\mathrm{y}$, si bien las medianas en ningún caso son equivalentes a dichas puntuaciones, sí son muy cercanas. Es importante destacar que la moda corresponde a la puntuación natural máxima en cada una de las distintas dimensiones (Véase Tabla 3).

$\mathrm{Al}$ igual que el IMC, en todas las dimensiones la asimetría es negativa, lo cual refuerza la apreciación respecto a la tendencia de los evaluados hacia las puntuaciones altas. Se presenta un comportamiento leptocúrtico en las distintas dimensiones lo que, en conjunto con la desviación, hace evidente la tendencia a la homogeneidad de las distribuciones. Tales comportamientos pueden deberse a un sesgo identificado con la deseabilidad por parte de los evaluados o un déficit en la capacidad de discriminación de la prueba.

En la Tabla 4, se muestra la correlación de las variables que componen los instrumentos a excepción de la variable resistencia al cambio. 
Tabla 4.

Correlaciones aceptación al cambio y calidad de vida laboral

\begin{tabular}{|c|c|c|c|c|c|c|c|c|}
\hline Variables & $\begin{array}{c}\text { Compen- } \\
\text { sación y } \\
\text { beneficios }\end{array}$ & $\begin{array}{c}\text { Condiciones } \\
\text { del ambi- } \\
\text { ente y del } \\
\text { trabajo }\end{array}$ & $\begin{array}{l}\text { Naturaleza } \\
\text { de la Tarea }\end{array}$ & $\begin{array}{c}\text { Desarrollo } \\
\text { y Seguridad } \\
\text { Laboral }\end{array}$ & $\begin{array}{l}\text { Democra- } \\
\text { cia Orga- } \\
\text { nizacional }\end{array}$ & $\begin{array}{l}\text { Derechos } \\
\text { Fundamen- } \\
\text { tales }\end{array}$ & $\begin{array}{c}\text { Equilibrio } \\
\text { Laboral }\end{array}$ & $\begin{array}{c}\text { Impacto } \\
\text { social }\end{array}$ \\
\hline Adaptabilidad & $0,718^{* *}$ & $0,576^{* *}$ & $0,616^{* *}$ & $0,638^{* *}$ & $0,665^{* *}$ & $0,568^{* *}$ & $0,584 * *$ & $0,567 * *$ \\
\hline Aceptación & $0,569^{* *}$ & $0,610^{* *}$ & $0,510^{* *}$ & $0,598^{* *}$ & $0,639 * *$ & $0,548^{* *}$ & $0,545^{* *}$ & $0,488^{* *}$ \\
\hline Comunicación & $0,521 * *$ & $0,567^{* *}$ & $0,581 * *$ & $0,548^{* *}$ & $0,571^{* *}$ & $0,594 * *$ & $0,524 * *$ & $0,573 * *$ \\
\hline $\begin{array}{l}\text { Conocimiento de } \\
\text { la organización }\end{array}$ & $0,497^{* *}$ & $0,588^{* *}$ & $0,565^{* *}$ & $0,512 * *$ & $0,496^{* *}$ & $0,467 * *$ & $0,567^{* *}$ & $0,500 * *$ \\
\hline $\begin{array}{l}\text { Cultura de } \\
\text { cambio }\end{array}$ & $0,584^{* *}$ & $0,593 * *$ & $0,568 * *$ & $0,594 * *$ & $0,570^{* *}$ & $0,554 * *$ & $0,633^{* *}$ & $0,543 * *$ \\
\hline $\begin{array}{l}\text { Gestión del } \\
\text { cambio (Factor } \\
\text { de segundo } \\
\text { Orden) }\end{array}$ & $0,538^{* *}$ & $0,622 * *$ & $0,621^{* *}$ & $0,556^{* *}$ & $0,608^{* *}$ & $0,543 * *$ & $0,505^{* *}$ & $0,630 * *$ \\
\hline Liderazgo & $0,550^{* *}$ & $0,567^{* *}$ & $0,619 * *$ & $0,612 * *$ & $0,633^{* *}$ & $0,560^{* *}$ & $0,537 * *$ & $0,613 * *$ \\
\hline Nivel Grupal & $0,499 * *$ & $0,614^{* *}$ & $0,630^{* *}$ & $0,541^{* *}$ & $0,593 * *$ & $0,615^{* *}$ & $0,509 * *$ & $0,635^{* *}$ \\
\hline Nivel Individual & $0,650^{* *}$ & $0,621^{* *}$ & $0,664 * *$ & $0,674 * *$ & $0,679 * *$ & $0,593 * *$ & $0,602 * *$ & $0,645^{* *}$ \\
\hline $\begin{array}{l}\text { Nivel } \\
\text { Organizacional }\end{array}$ & $0,448^{* *}$ & $0,400^{* *}$ & $0,319 * *$ & $0,432 * *$ & $0,433 * *$ & $0,448^{* *}$ & $0,474 * *$ & $0,336 * *$ \\
\hline $\begin{array}{l}\text { Participación } \\
\text { Integración }\end{array}$ & $0,377^{* *}$ & $0,474 * *$ & $0,459 * *$ & $0,360^{* *}$ & $0,426^{* *}$ & $0,374 * *$ & $0,329^{* *}$ & $0,474 * *$ \\
\hline $\begin{array}{l}\text { Proyección } \\
\text { y desarrollo } \\
\text { del cambio } \\
\text { (Segundo Orden) }\end{array}$ & $0,573^{* *}$ & $0,607^{* *}$ & $0,645^{* *}$ & $0,567^{* *}$ & $0,618^{* *}$ & $0,620^{* *}$ & $0,600^{* *}$ & $0,610^{* *}$ \\
\hline $\begin{array}{l}\text { Resistencia al } \\
\text { cambio }\end{array}$ & $-0,061$ & $-0,154$ & $-0,166$ & $-0,098$ & $-0,058$ & $-0,054$ & $-0,049$ & $-0,086$ \\
\hline $\begin{array}{l}\text { Trabajo en } \\
\text { equipo }\end{array}$ & $0,433 * *$ & $0,527^{* *}$ & $0,609 * *$ & $0,511^{* *}$ & $0,545^{* *}$ & $0,609 * *$ & $0,515^{* *}$ & $0,599 * *$ \\
\hline
\end{tabular}

Nota: $* *$ Significancia del 0,01 .

La variable adaptabilidad del IMC correlaciona al 0.01 con las variables del instrumento de calidad de vida laboral: compensación y beneficios ( $\mathrm{Rho}=0,718)$; condiciones del ambiente de trabajo $(\mathrm{Rho}=0,576)$; naturaleza de la tarea ( $\mathrm{Rho}=0,616)$; desarrollo y seguridad laboral $(\mathrm{Rho}=0,638)$; democracia organizacional $(\mathrm{Rho}=0,665)$; derechos fundamentales $(\mathrm{Rho}=0,568)$; equilibrio laboral $(\mathrm{Rho}=0,584)$; e impacto social $(\mathrm{Rho}=0,567)$.

La variable aceptación del IMC correlaciona al 0.01 con las variables del instrumento de calidad de vida laboral: compensación y beneficios $(\mathrm{Rho}=0,569)$; condiciones del ambiente de trabajo $(\mathrm{Rho}=0,610)$; naturaleza de la tarea $(\mathrm{Rho}=0,510)$; desarrollo y seguridad laboral $(\mathrm{Rho}=0,598)$; democracia organizacional $(\mathrm{Rho}=0,639)$; derechos fundamentales $(\mathrm{Rho}=0,548)$; equilibrio laboral $(\mathrm{Rho}=0,545)$; e impacto social $(\mathrm{Rho}=0,488)$.

La variable de comunicación del IMC correlaciona al 0.01 con las variables del instrumento de calidad de vida laboral: compensación y beneficios $(\mathrm{Rho}=0,521)$; condiciones del ambiente de trabajo (Rho=0,567); naturaleza de la tarea $(\mathrm{Rho}=0,581)$; desarrollo y seguridad laboral $(\mathrm{Rho}=0,548)$; democracia organizacional $(\mathrm{Rho}=0,571)$; 
derechos fundamentales $(\mathrm{Rho}=0,594)$; equilibrio laboral (Rho=0,524); e impacto social $(\mathrm{Rho}=0,573)$.

La variable de conocimiento de la organización del IMC correlaciona al 0.01 con las variables del instrumento de calidad de vida laboral: compensación y beneficios $(\mathrm{Rho}=0,497)$; condiciones del ambiente de trabajo $(\mathrm{Rho}=0,588)$; naturaleza de la tarea $(\mathrm{Rho}=0,565)$; desarrollo y seguridad laboral $(\mathrm{Rho}=0,512)$; democracia organizacional $(\mathrm{Rho}=0,496)$; derechos fundamentales $(\mathrm{Rho}=0,467)$; equilibrio laboral $(\mathrm{Rho}=0,567)$; e impacto social $(\mathrm{Rho}=0,500)$.

La variable de cultura de cambio del IMC correlaciona al 0.01 con las variables del instrumento de calidad de vida laboral: compensación y beneficios $(\mathrm{Rho}=0,584)$; condiciones del ambiente de trabajo $(\mathrm{Rho}=0,593)$; naturaleza de la tarea $(\mathrm{Rho}=0,568)$; desarrollo y seguridad laboral $(\mathrm{Rho}=0,594)$; democracia organizacional $(\mathrm{Rho}=0,570)$; derechos fundamentales $(\mathrm{Rho}=0,554)$; equilibrio laboral $(\mathrm{Rho}=0,633)$; e impacto social $(\mathrm{Rho}=0,543)$.

La variable de gestión del cambio del IMC correlaciona al 0.01 con las variables del instrumento de calidad de vida laboral: compensación y beneficios $(\mathrm{Rho}=0,538)$; condiciones del ambiente de trabajo $(\mathrm{Rho}=0,622)$; naturaleza de la tarea $(\mathrm{Rho}=0,621)$; desarrollo y seguridad laboral $(\mathrm{Rho}=0,556)$; democracia organizacional $(\mathrm{Rho}=0,608)$; derechos fundamentales ( $\mathrm{Rho}=0,543)$; equilibrio laboral ( Rho=0,505); e impacto social $(\mathrm{Rho}=0,630)$.

La variable de liderazgo del IMC correlaciona al 0.01 con las variables del instrumento de calidad de vida laboral: compensación y beneficios $(\mathrm{Rho}=0,550)$; condiciones del ambiente de trabajo $(\mathrm{Rho}=0,567)$; naturaleza de la tarea $(\mathrm{Rho}=0,619)$; desarrollo y seguridad laboral $(\mathrm{Rho}=0,612)$; democracia organizacional $(\mathrm{Rho}=0,633)$; derechos fundamentales $(\mathrm{Rho}=0,560)$; equilibrio laboral $(\mathrm{Rho}=0,537)$; e impacto social $(\mathrm{Rho}=0,613)$.

La variable de nivel grupal del IMC correlaciona al 0.01 con las variables del instrumento de calidad de vida laboral: compensación y beneficios ( $\mathrm{Rho}=0,499)$; condiciones del ambiente de trabajo $(\mathrm{Rho}=0,614)$; naturaleza de la tarea $(\mathrm{Rho}=0,630)$; desarrollo y seguridad laboral $(\mathrm{Rho}=0,541)$; democracia organizacional $(\mathrm{Rho}=0,593)$; derechos fundamentales $(\mathrm{Rho}=0,615)$; equilibrio laboral $(\mathrm{Rho}=0,509)$; e impacto social $(\mathrm{Rho}=0,635)$.

La variable de nivel individual del IMC correlaciona al 0.01 con las variables del instrumento de calidad de vida laboral: compensación y beneficios $(\mathrm{Rho}=0,650)$; condiciones del ambiente de trabajo ( $\mathrm{Rho}=0,621)$; naturaleza de la tarea $(\mathrm{Rho}=0,664)$; desarrollo y seguridad laboral $(\mathrm{Rho}=0,674)$; democracia organizacional $(\mathrm{Rho}=0,679)$; derechos fundamentales ( $\mathrm{Rho}=0,593)$; equilibrio laboral $(\mathrm{Rho}=0,602)$; e impacto social $(\mathrm{Rho}=0,645)$.
La variable de nivel organizacional del IMC correlaciona al 0.01 con las variables del instrumento de calidad de vida laboral: compensación y beneficios $(\mathrm{Rho}=0,448)$; condiciones del ambiente de trabajo $(\mathrm{Rho}=0,400)$; naturaleza de la tarea $(\mathrm{Rho}=0,319)$; desarrollo y seguridad laboral $(\mathrm{Rho}=0,432)$; democracia organizacional $(\mathrm{Rho}=0,433)$; derechos fundamentales $(\mathrm{Rho}=0,448)$; equilibrio laboral $(\mathrm{Rho}=0,474)$; e impacto social $(\mathrm{Rho}=0,336)$.

La variable de participación e integración del IMC correlaciona al 0.01 con las variables del instrumento de calidad de vida laboral: compensación y beneficios $(\mathrm{Rho}=0,377)$; condiciones del ambiente de trabajo $(\mathrm{Rho}=0,474)$; naturaleza de la tarea $(\mathrm{Rho}=0,459)$; desarrollo y seguridad laboral $(\mathrm{Rho}=0,360)$; democracia organizacional $(\mathrm{Rho}=0,426)$; derechos fundamentales $(\mathrm{Rho}=0,374)$; equilibrio laboral $(\mathrm{Rho}=0,329)$; e impacto social $(\mathrm{Rho}=0,474)$.

En cuanto a la variable de proyección y desarrollo del cambio del IMC, correlaciona al 0.01 con las variables del instrumento de calidad de vida laboral: compensación y beneficios $(\mathrm{Rho}=0,573)$; condiciones del ambiente de trabajo $(\mathrm{Rho}=0,607)$; naturaleza de la tarea $(\mathrm{Rho}=0,645)$; desarrollo y seguridad laboral $(\mathrm{Rho}=0,567)$; democracia organizacional $(\mathrm{Rho}=0,618)$; derechos fundamentales $(\mathrm{Rho}=0,620)$; equilibrio laboral $(\mathrm{Rho}=0,600)$; e impacto social $(\mathrm{Rho}=0,610)$.

La variable de trabajo en equipo del IMC correlaciona al 0.01 con las variables del instrumento de calidad de vida laboral: compensación y beneficios $(\mathrm{Rho}=0,433)$; condiciones del ambiente de trabajo $(\mathrm{Rho}=0,527)$; naturaleza de la tarea $(\mathrm{Rho}=0,609)$; desarrollo y seguridad laboral $(\mathrm{Rho}=0,511)$; democracia organizacional $(\mathrm{Rho}=0,545)$; derechos fundamentales ( $\mathrm{Rho}=0,609)$; equilibrio laboral (Rho=0,515); e impacto social $(\mathrm{Rho}=0,599)$.

\section{DISCUSIÓN}

El objetivo general de esta investigación fue identificar la relación entre calidad de vida laboral y la disposición al cambio organizacional en los funcionarios de una entidad pública de la ciudad de Bogotá.

Para dar respuesta al primer objetivo específico, se identificó la disposición de los trabajadores frente al cambio. Los resultados arrojados muestran una tendencia positiva en todas las variables y factores evaluados con el IMC. Sin embargo, es necesario hacer dos comentarios: el primero tiene que ver con el sesgo evidente de la muestra hacia los valores máximos de la prueba, mucho más evidente en las variables Adaptabilidad, Cultura de cambio, Liderazgo y Participación / Integración; así como en los factores Individual, Gestión del cambio y Proyección y Desarrollo 
del cambio. Y el segundo está relacionado con la asimetría y curtosis que evidencian no solo una tendencia hacia los valores altos, sino hacia una disposición más bien homogénea de la distribución.

En relación con el primer aspecto, es necesario tener en consideración los factores motivacionales y las percepciones respecto a las posibles implicaciones de los miembros de la muestra frente a la investigación. Es claro que por consideraciones éticas, la participación es estrictamente anónima y de carácter confidencial, pero esa no es necesariamente la consideración que tienen muchos de los participantes en este tipo de investigaciones.

En segundo lugar, a pesar de las condiciones técnicas bajo las cuales se diseñó la prueba y que evidencian adecuados niveles de confiabilidad, resulta conveniente cuestionar la calidad y la sensibilidad para la medición de las variables que se supone son evaluadas con la misma. Es decir, se requeriría un análisis técnico de fondo para poder determinar si la prueba cumple con las condiciones necesarias requeridas para una evaluación sensible a los aspectos que se propone evaluar.

En cuanto al segundo objetivo, el cual pretendió identificar las condiciones de calidad de vida, se encontró una alta valoración por parte de los evaluados, dando lugar a promedios, medianas y modas ubicados en los extremos superiores de cada una de las dimensiones. Sin embargo, al igual que con la medición del cambio, es conveniente cuestionar la capacidad de la prueba para valorar adecuadamente la variable que se supone está midiendo, y los sesgos generados por los evaluados como resultantes de juicios personales o creencias inadecuadas respecto a los alcances y limitaciones éticas de la investigación.

Es claro, en ambos casos, que otras variables pueden incidir en las respuestas de los evaluados a este tipo de pruebas. Aspectos como la motivación, la ansiedad, las dudas sobre los efectos posteriores que pueda tener la socialización de los resultados, entre otros, son elementos que pueden afectar la autenticidad del evaluado al momento de responder.

Para dar respuesta al objetivo general, se identificó a partir del análisis de resultados, que la mayoría de variables de cambio organizacional correlacionan con las variables del instrumento de calidad de vida laboral, con la excepción de la variable de resistencia al cambio. Al respecto, Robbis (2002) menciona cinco razones por las cuales cree que las organizaciones se resisten al cambio: la costumbre, la seguridad, los factores económicos, el procesamiento selectivo y la selección de la información de lo que escuchan, Esto puede explicar que la resistencia al cambio no solo se debe a una situación actual de la entidad referente a la calidad de vida laboral, sino que otros factores pueden llegar a intervenir, mejorando así la productividad y la funcionalidad en el trabajo realizado por cada uno de los empleados (Dolan \& García - Sánchez, 1999).

Teniendo en cuenta que las variables de los dos instrumentos aplicados correlacionan de manera significativa, se puede señalar que la calidad de vida dentro del ambiente de trabajo y los beneficios dados por la entidad abren paso a que el empleado se adapte adecuadamente a los cambios que se producen dentro de la organización. Al respecto, de la Poza y Prior (1988, citados por González, Peiró \& Bravo, 1996), explican cuáles son los factores que dan cuenta de la forma en que se produce la experiencia laboral, tanto en sus condiciones objetivas (seguridad, higiene salario, etc.), como en sus condiciones subjetivas (la forma como vive el trabajador).

Es importante resaltar estos resultados, ya que no solo evidencian la relación de las variables objeto de estudio, sino que también permiten aceptar la hipótesis de investigación. Adicionalmente, se puede establecer que la calidad de vida constituye una percepción subjetiva del cambio organizacional.

En este orden de ideas, así como el cambio organizacional puede ser estudiado desde diferentes perspectivas del comportamiento organizacional, la calidad de vida también se puede abordar desde diferentes puntos de vista; en este caso, desde la óptica del trabajador, la cual se constituye en un elemento subjetivo. De igual forma, cuando se estudia desde aspectos psicológicos, en donde se consideran características individuales, como las características emocionales y actitudinales, también se evidencian aspectos subjetivos (Segurado \& Argulló, 2000).

Por lo anterior, los aspectos psicológicos del individuo, que se han estudiado con el cambio organizacional, tales como la actitud (García, Rojas \& Díaz, 2011), la motivación (García, Gómez \& Londoño, 2009), la motivación y satisfacción (García \& Forero, 2014), y la justicia organizacional (García \& Forero, 2014), constituyen elementos importantes para concluir que las percepciones del trabajador sobre diferentes procesos de cambio van a depender de la manera como los vivió y comprendió desde su punto de vista.

Adicionalmente, se puede establecer que tanto el cambio organizacional como la calidad de vida laboral son multidimensionales; por lo tanto, no se pueden entender como elementos inarticulados en las organizaciones y, por el contrario, van a estar afectados por otros aspectos que de alguna manera están generando alguna influencia en la forma como los trabajadores las perciben.

El impacto social que tendrá esta investigación será la generación de más aportes teóricos importantes para la psi- 
cología organizacional con respecto a la relación existente entre las variables mencionadas, así como la posibilidad de generar más teoría a nivel empírico por medio de la revisión de la literatura existente sobre dichas variables. Por otra parte, puede contribuir a la investigación sobre nuevas relaciones y propiciar explicaciones que aporten a los organizaciones colombianas la forma como se maneja un cambio adecuado teniendo en cuenta algunas variables del individuo.

\section{REFERENCIAS}

Acosta, C. (2004) Cuatro preguntas para iniciarse en cambio organizacional. Revista Colombiana de Psicología, 11, 9-24.

Alcover, M., Rico, R., \& Gil, F. (2011). Equipos de trabajo en contextos organizacionales: Dinámicas de cambio, adaptación y aprendizaje en entornos flexibles. Papeles de psicó$\log$ o, 32, 7-16.

Arrieiro, C., \& Natividade, L. (2009), Qualidade de vida no trabalho. Revista Tecer Belo Horizont, 2, 1-12.

Camacaro, P. (2010). Aproximación a la calidad de vida en el trabajo en la organización castrense venezolana. Edición electrónica. Acosta, C. (2004) Cuatro preguntas para iniciarse en cambio organizacional. Revista Colombiana de Psicología, 11, 9-24.

Cañon, S., \& Galeano, G. (2011). Factores laborales psicosociales y calidad de vida laboral de los trabajadores de la salud de ASSBASALUD E.S.E. Archivos de Medicina. 11 (2), 114 - 126.

Chiang M.M., \& Krausse, K (2009). Estudio empírico de calidad de vida laboral, cuatro indicadores: satisfacción laboral, condiciones y medioambiente del trabajo, organización e indicador global, sectores privado y público. Horizontes empresariales. 23-50.

Coolican, H. (2005). Métodos de investigación y estadística en psicología (3ra Ed.) México: Manual Moderno.

Da Silva, M., (2006). Nuevas perspectivas de la calidad de vida laboral y sus relaciones con la eficacia organizacional. Tesis doctoral: Universidad de Barcelona.

Díaz, J. (2005) Cambio organizacional, una aproximación por valores. Revista Venezolana de Gerencia. 10(32), 605-627.

Duran, M. (2010). Bienestar psicológico: el estrés y la calidad de vida en el contexto laboral. $R N A$ revista nacional de administración, 1, 71-84.

Dolan, S.L., \& García-Sánchez, S. (1999), La gestion par valeurs. Montréal: Éditions Nouvelles.

Duro, A. (2004). Calidad de vida laboral. Calidad de vida laboral: Área de Psicología Social. España: Universidad Rey Juan Carlos.

Elizur, D. et Shye, S. (1990). «Quality of work life and its relation to quality of life». Applied Psychology: An International Review, 39(3), 275-291
Ernst, E., Lewis, S., \& Hammer, L (2010). Work-life initiatives and organizational change: Overcoming mixed messages to move from the margin to the mainstream. Hum Relat, 63(1), 3-19.

Ferreira, D., Barbosa, I., Hastenreiter, J.M., \& De Acevedo, F. (2011), Mudança organizacional em uma instituição hospitalar: um estudo de caso sobre as percepções dos gestores. Belo Horizonte. 12(2), 85-110

Flores, N., Jenaro, C., González, F., \& García P. (2010) Análisis de la calidad de vida laboral en trabajadores con discapacidad. Instituto Universitario de Integración en la Comunidad (INICO), Universidad de Salamanca. Pp. 95-107.

Freire, J., \& Gutiérrez-Rubí, A (2010). 32 tendencias de cambio. España: Gráfico.

García M., Rojas M., \& Díaz S. (2011). Relación entre el cambio organizacional y la actitud al cambio en trabajadores de una empresa de Bogotá. Diversitas Perspectivas en Psicología, (1) 7, 2-1

García, G., \& Sandoval, A. (2010), Cultura gerencial y cambio organizacional en Cierres Ideal de México: un "modelo" de calidad guadalupano. Revista Redalyc, 34, 133-148.

García, M, Gómez, G y Londoño, O. (2009). Relación entre motivación y resistencia al cambio en personas que trabajan en una empresa del sector público. Revista Diversitas Perspectivas en Psicología, 5(1), 141-159.

García, M. \& Forero, C. (2010). IMC - Instrumento de Medición de Cambio Organizacional. Manual Técnico. Bogotá, Colombia: RH\&T. (Documento no publicado).

García, M. (2011). Liderazgo transformacional y la facilitación de la aceptación al cambio organizacional. Pensamiento Psicológico, 9(16), 41-54.

García, M., \& Forero, C. (2014). Cambio organizacional y percepción de la justicia organizacional en una entidad de la ciudad de Bogotá-Colombia. Revista Diversitas Perspectivas en Psicología. 10(2), 293 -305

García, M., \& Forero, C. (2014). Motivación y satisfacción laboral como facilitadores del cambio organizacional: una explicación desde las ecuaciones estructurales. Psicogente, 17(31), 120-142.

García, M., Arias, F., \& Gómez, P. (2013). Relación entre comunicación y cambio organizacional en trabajadores de una empresa del sector terciario. Revista Diversitas Perspectivas en Psicología. 9(1), 89-95.

García, M., Camarco, J., Cervera, A., Ramírez, A \& Romero, B. (2010). Relación entre grupos/ equipos de trabajo y cambio organizacional en una empresa comercializadora de Bogotá. Psychologia: avances de la disciplina. 4(2), 31-46.

Giraldo, M (2009). Modelo de gestión del cambio planeado para la sunción supervisoria, Caso empresa de consumo masivo, 3rd, International, Conference on Industrial Engineering and Industrial Management, XII Congreso de Organizaciones e Ingeniería, Barcelona - Terrassa, Sept. 2-4. 
Gómez, M.A. (2010). Calidad de vida laboral en empleados temporales del Valle de Aburrá - Colombia. Revista Ciencias Estratégica, 18(24), 225-236.

Gómez-Rada, C (2011). Instrumento calidad de vida laboral. Manual Técnico. Bogotá, Colombia. Documento no publicado.

González, P., Peiró, J.M., \& Bravo, M. (1996). Calidad de vida laboral. In J.M.Peiró \& F.Prieto (Eds.): Tratado de Psicología del Trabajo (vol. 2). Madrid: Síntesis (pp. 161-186).

González, T. (2010). Agroindustrias: cambio organizacional como estrategias de sobrevivencia y transformación regional. Universidad autónoma, 4, 1-12.

Granados, I. (2011) Calidad de vida laboral: historia, dimensiones y beneficios. Revista IIPSI. 14(2), 271-276

Hernández, P., Gallarzo, M., \& Espinosa, J. (2011). Desarrollo organizacional enfoque latinoamericano. México: Pearson.

Hernández, R., Fernández, C., \& Baptista, P. (2010). Metodología de la Investigación. México: McGraw Hill.

Jeanie, D. (2002). El monstruo del cambio, El factor humano como elemento decisivo para estimular o frustrar el cambio en la empresa. España, Ediciones Urano S.A.

Kerber, K., \& Buono, A. (2010). Intervention and organizational change: building organizational change capacity. En Buono, A.F. \& Jamieson, D.W. (Eds.).

Kotter, P., \& Cohen, D. (2003). Las claves del cambio. España: Eusto.

Luecke, R. (2003). Gestionar el cambio y la transición. España: Deusto.

Montalván, C., (2005). Calidad de vida laboral: hacia un enfoque integrador desde la Psicologia Social. España: Universidad de Oviedo.

Nair, S. (2013). A study on the effect of quality of work life (QWL) on organizational citizenship behaviour (OCB) With Special reference to College Teachers is Thrissur District, Kerala . Integral Review-A Journal of Management. 6(1), 34-46.
Ooi, T. (2011). Organization Transformation - What matters most is the Leader's Actions. Int. J. Emerg. Sci. 1(1), 211-230

Palací, F.J. (2005). Psicología de la Organización. España: Pearson-Prentice Hall.

Palací, F.J. (2005). Psicología de la Organización. España: Pearson-Prentice Hall.

Pérez, C. (2001). Técnicas estadísticas con SPSS. Madid, España: Prentice - Hall

Pérez, T., Campos, A., Negro, A., \& Caballero, F. (2011). Professional Burnout and Work Satisfaction In Spanish Allergists: Analysis of Working Conditions in the Specialty. $J$. Investing Allergol. Clin. Immunol. 21(1), 13-21.

Quirant, E. (2006). La importancia del factor humano para lograr el éxito del proceso de cambio. Revista de empresa. 18, 50-63.

Robbins, S. (2004). Comportamiento Organizacional, México, Editorial: Pearson.

Ruiz,M., Ruiz, S., Martínez, L., \& Peláez, I. (1999). Modelo para la gestión del cambio organizacional en las pymes. Departamento de Economía de la Empresa. Universidad de Murcia.

Segurado, A., \& Agulló, E. (2002). Calidad de vida laboral: hacia un enfoque integrador desde la psicología social. Psicothema. 14(4) 828 - 836.

Toro, F. (1991). Desempeño y productividad. Contribuciones de la psicología organizacional. Medellín: Cincel

Uribe - Rodríguez, A. F., Garrido - Pinzón, J., \& Rodríguez, A.M (2011). Influencia del tipo de contratación en la calidad de vida laboral: manifestaciones del capitalismo organizacional. Revista Virtual Universidad Católica del Norte. $33,101-116$.

Vincenzi, L., Melo, M., \& Fonseca, P. (2011) Resistência À Mudança Organizacional: Avaliação de Atitudes e Reações em Grupo de Indivíduos. 1-15. 